\title{
Structure Design And Analysis Of Kinematics Of An Upper- limbed Rehabilitation Robot
}

\author{
Zhiming Wang ${ }^{1}$, Zhenglong Cai ${ }^{1, a}$, Lizheng Cui ${ }^{1}$, Changfu Pang ${ }^{1}$ \\ ${ }^{1}$ School of Mechatronic Engineering and Automation, Shanghai University, Shanghai 200072, China
}

\begin{abstract}
According to the physiological structure of human upper limb, a 5-DOF upper-limbed rehabilitation robot is designed in this paper. Based on the software of Pro/E, the robot model is established. Based on the theory of robot coordinate transformation, the equations of motion of robot are established by the method of D-H. Three dimensional model of the rehabilitation robot is imported into the software of ADAMS for the kinematics analysis and simulation. The obtained simulation position curves are consistent with the theoretical position curves, verifying the correctness of the theoretical derivation. The result of the simulation proves the correctness of the kinematics equation. These studies provide an important basis for the realization of mechanism control.
\end{abstract}

\section{Introduction}

Hemiplegia is one of the major physical dysfunctions caused by stroke and brain trauma. The recovery of upper limb dysfunction is slower than lower limb dysfunction, which will delay the recovery process of hemiplegia. Therefore, it is important to find effective rehabilitation therapy to improve the upper limb function of hemiplegic patients. Medical theory has proved that correct and scientific rehabilitation training is helpful to the recovery and improvement of limb motion function in addition to surgery and drug therapy [1]. In this paper, a 5-DOF upper limb rehabilitation robot is designed, according to the physiological and kinematic characteristics of human upper limb.

Upper limb rehabilitation robot assists hemiplegic patients in rehabilitation training mainly through the following four training methods: passive rehabilitation training, active rehabilitation training and impedance rehabilitation training [2]. In the early stage of rehabilitation, the upper limb rehabilitation robot drives the upper limb to train passively according to the planned trajectory. In the late rehabilitation stage, the upper limb rehabilitation robot recognizes the movement intention of the patient according to the surface electromyography signal, and assists the patient to complete the active rehabilitation training. Impedance rehabilitation training is intended to enhance training effect.

\section{Physiological analysis of human upper-limb}

The human upper-limb bones are composed of collarbone, scapula, humerus, ulna, radius, wrist, metacarpal and phalanx [3], as shown in Figure 1. The upper limb can be divided into two parts: arm and hand. The arm is divided into the upper arm and the forearm. There are more skeletal joints in the hand. The finger movement is not considered in this paper. As shown in Figure 2, shoulder joint has three degrees of freedom: horizontal and vertical movement, flexion and extension movement, internal and external rotation. Elbow joint has one degree of freedom for flexion and extension. The forearm has one degree of freedom for internal and external rotation. The wrist joint has two degrees of freedom: flexion and extension movement, radial and ulnar deviation movement. Considering that the upperlimb rehabilitation robot is applied to patients with hemiplegia, the joint activity of the rehabilitation action should be less than the joint activity of the healthy person at the time of design.

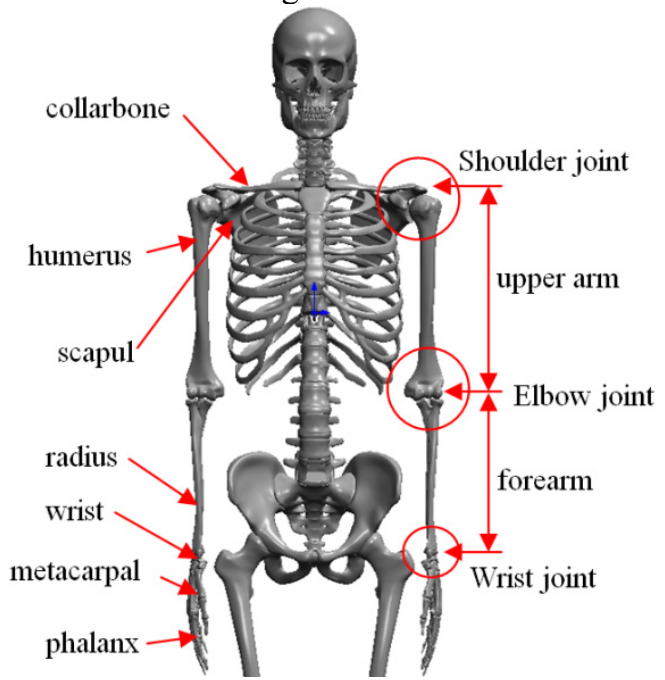

Figure 1. Upper-limb skeleton structure.

*Corresponding author: ${ }^{\text {a }}$ Zhenglong Cai:czlongal@163.com 


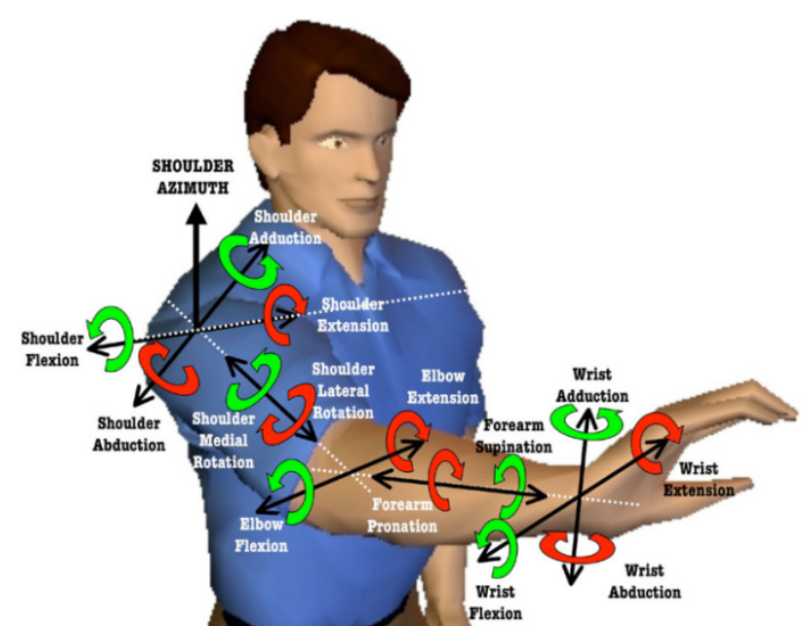

Figure 2. The seven principal degrees of freedom of the human arm(adapted from [4]).

\section{Structural design of rehabilitation robot}

Due to the seven degrees of freedom of the upper limb of the human body, the complexity of design and manufacture, manufacturing cost and the amount of control calculation of the upper limb rehabilitation robot are greatly increased. In order to ensure the cost of the organization and the lightness of the structure, the degree of freedom in the upper-limb robot model is chosen in the design.

In summary, the simplified upper-limb model includes five degrees of freedom: horizontal and vertical movement, flexion and extension movement, internal and external rotation of shoulder joint, flexion and extension of elbow joint, flexion and extension movement of wrist joint. All joints of the upper limb rehabilitation robot are rotating joints, and each joint is equipped with motor, reducer and angle sensor. As shown in Figure 3, the robot has a lifting platform. As shown in Figure 4, the length of the upper arm, forearm and palm of robot can be adjusted. It is suitable for people with different height and arm length.

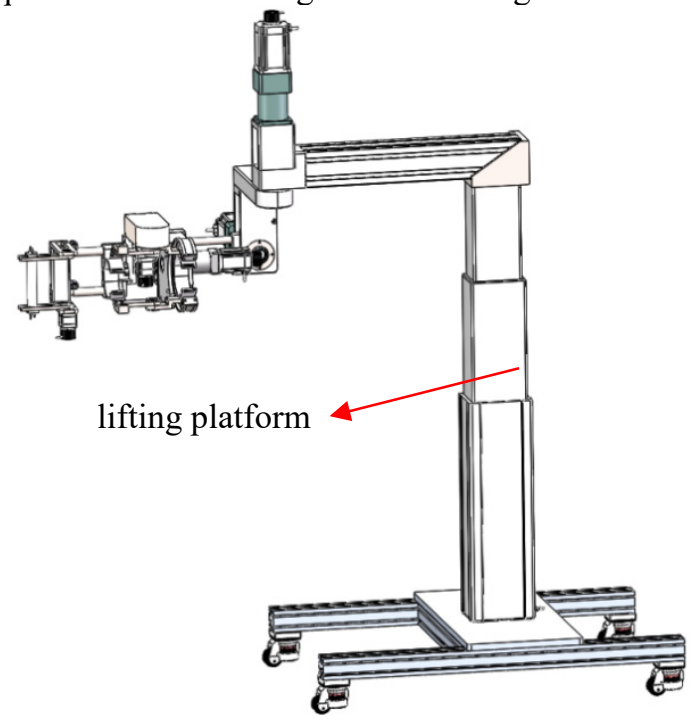

Figure 3. The upper-limb rehabilitation robot.

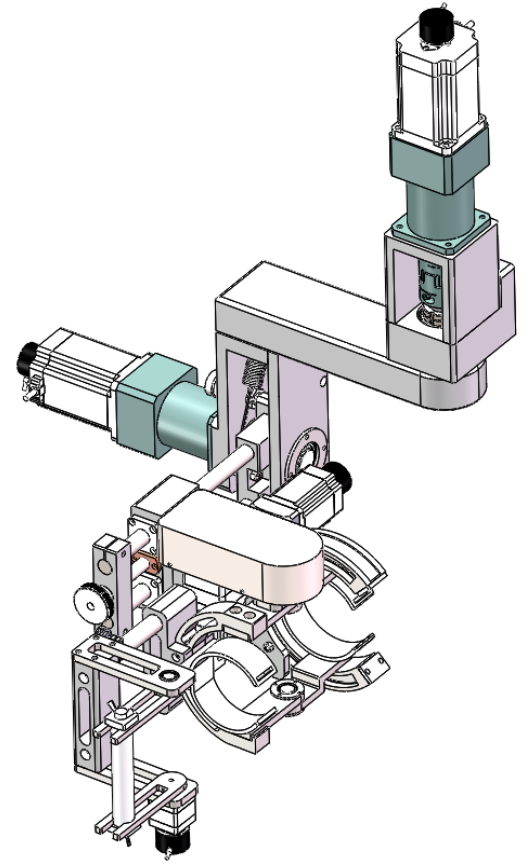

Figure 4. Structure of the upper-limb rehabilitation robot.

\section{Kinematic analysis}

Kinematics analysis of rehabilitation robot is the theoretical basis of robot motion [5]. Kinematic analysis includes positive kinematics solution and inverse kinematics solution. The positive solution is to get the pose of the robot through the variation of angle of the robot joint. The inverse solution is to get the angle of robot joints through terminal pose. This relationship is analyzed by Cartesian coordinate transformation. According to the establishment rule of D-H coordinate system, we simplify the model and establish the D-H coordinate system of upper limb rehabilitation robot. The coordinate system is shown in Figure 5. According to the $\mathrm{D}-\mathrm{H}$ matrix parameter definition rule, the $\mathrm{D}-\mathrm{H}$ matrix parameter value shown in Table 1 is gained.

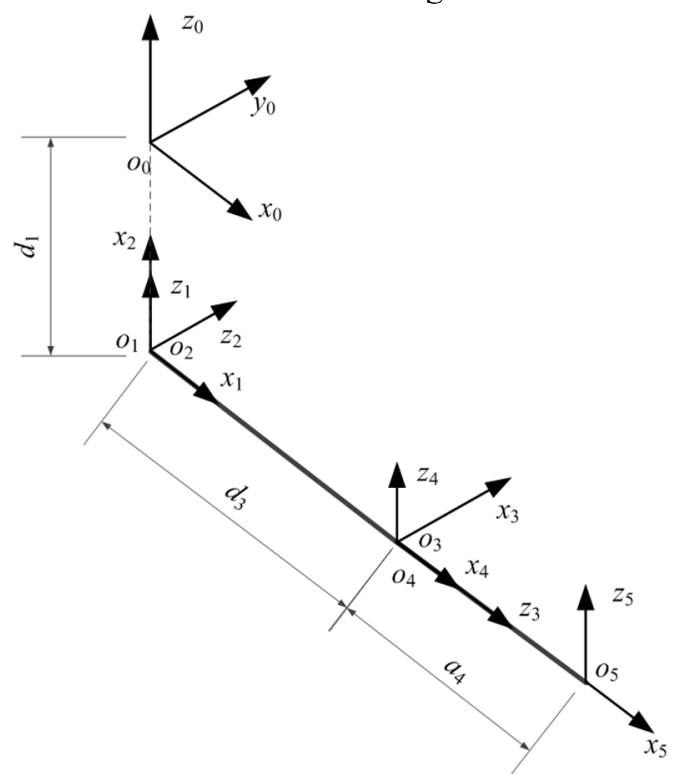

Figure 5. D-H parameter system of the upper-limb rehabilitation robot. 
Table 1. D-H parameters of 5-DOF upper-limb rehabilitation

\begin{tabular}{|c|c|c|c|c|}
\hline$i$ & $\alpha_{i-1}$ & $a_{i-1}(m)$ & $d_{i}(m)$ & $\theta_{i}$ \\
\hline 1 & $0^{\circ}$ & 0 & $-d_{1}$ & $\theta_{1}\left(0^{\circ}\right)$ \\
\hline 2 & $-90^{\circ}$ & 0 & 0 & $\theta_{2}\left(-90^{\circ}\right)$ \\
\hline 3 & $-90^{\circ}$ & 0 & $d_{3}$ & $\theta_{3}\left(-90^{\circ}\right)$ \\
\hline 4 & $-90^{\circ}$ & 0 & 0 & $\theta_{4}\left(-90^{\circ}\right)$ \\
\hline 5 & $0^{\circ}$ & $a_{4}$ & 0 & $\theta_{5}\left(0^{\circ}\right)$ \\
\hline
\end{tabular}

By substituting the above parameters into the D-H homogeneous transformation matrix, the transformation matrix of each link can be obtained as follows:

$$
\begin{aligned}
& { }_{1} T=\left[\begin{array}{cccc}
c \theta_{1} & -s \theta_{1} & 0 & 0 \\
s \theta_{1} & c \theta_{1} & 0 & 0 \\
0 & 0 & 1 & -d_{1} \\
0 & 0 & 0 & 1
\end{array}\right],{ }_{2}^{1} T=\left[\begin{array}{cccc}
c \theta_{2} & -s \theta_{2} & 0 & 0 \\
0 & 0 & 1 & 0 \\
-s \theta_{2} & -c \theta_{2} & 0 & 0 \\
0 & 0 & 0 & 1
\end{array}\right] \\
& { }_{3}^{2} T=\left[\begin{array}{cccc}
c \theta_{3} & -s \theta_{3} & 0 & 0 \\
0 & 0 & 1 & d_{3} \\
-s \theta_{3} & -c \theta_{3} & 0 & 0 \\
0 & 0 & 0 & 1
\end{array}\right],{ }_{4}^{3} T=\left[\begin{array}{cccc}
c \theta_{4} & -s \theta_{4} & 0 & 0 \\
0 & 0 & 1 & 0 \\
-s \theta_{4} & -c \theta_{4} & 0 & 0 \\
0 & 0 & 0 & 1
\end{array}\right]
\end{aligned}
$$$$
{ }_{5}^{4} T=\left[\begin{array}{cccc}
c \theta_{5} & -s \theta_{5} & 0 & a_{4} \\
s \theta_{5} & c \theta_{5} & 0 & 0 \\
0 & 0 & 1 & 0 \\
0 & 0 & 0 & 1
\end{array}\right]
$$

The positive kinematics solution of the rehabilitation robot is as follows:

${ }_{5}^{0} T={ }_{1}^{0} T_{2}^{1} T_{3}^{2} T_{4}^{3} T_{5}^{4} T=\left[\begin{array}{cccc}n_{x} & o_{x} & a_{x} & p_{x} \\ n_{y} & o_{y} & a_{y} & p_{y} \\ n_{z} & o_{z} & a_{z} & p_{z} \\ 0 & 0 & 0 & 1\end{array}\right]$

$n_{x}=\mathrm{c}_{5}\left(\mathrm{c}_{4} \mathrm{~s}_{1}\left(\mathrm{~s}_{3}+\mathrm{c}_{1} \mathrm{c}_{2} \mathrm{c}_{3}\right)+\mathrm{c}_{1} \mathrm{~s}_{4} \mathrm{~s}_{2}\right)-$

$$
\mathrm{s}_{5}\left(\mathrm{~s}_{4}\left(\mathrm{~s}_{1} \mathrm{~s}_{3}+\mathrm{c}_{1} \mathrm{c}_{2} \mathrm{c}_{3}\right)-\mathrm{c}_{4} \mathrm{c}_{1} \mathrm{~s}_{2}\right)
$$

$n_{y}=\mathrm{s}_{5}\left(\mathrm{~s}_{4}\left(\mathrm{c}_{1} \mathrm{~s}_{3}-\mathrm{c}_{2} \mathrm{c}_{3} \mathrm{~s}_{1}\right)+\mathrm{c}_{4} \mathrm{~s}_{1} \mathrm{~s}_{2}\right)-$

$$
\mathrm{c}_{5}\left(\mathrm{c}_{4}\left(\mathrm{c}_{1} \mathrm{~s}_{3}-\mathrm{c}_{2} \mathrm{c}_{3} \mathrm{~s}_{1}\right)-\mathrm{s}_{4} \mathrm{~s}_{1} \mathrm{~s}_{2}\right)
$$

$n_{z}=\mathrm{c}_{5}\left(\mathrm{c}_{2} \mathrm{~s}_{4}-\mathrm{c}_{4} \mathrm{c}_{3} \mathrm{~s}_{2}\right)+\mathrm{s}_{5}\left(\mathrm{c}_{4} \mathrm{c}_{2}+\mathrm{c}_{3} \mathrm{~s}_{4} \mathrm{~s}_{2}\right)$

$o_{x}=-c_{5}\left(s_{4}\left(s_{1} s_{3}+c_{1} c_{2} c_{3}\right)-c_{4} c_{1} s_{2}\right)-$

$$
\mathrm{s}_{5}\left(\mathrm{c}_{4}\left(\mathrm{~s}_{1} \mathrm{~s}_{3}+\mathrm{c}_{1} \mathrm{c}_{2} \mathrm{c}_{3}\right)+\mathrm{c}_{1} \mathrm{~s}_{4} \mathrm{~s}_{2}\right)
$$

$o_{y}=\mathrm{c}_{5}\left(\mathrm{~s}_{4}\left(\mathrm{c}_{1} \mathrm{~s}_{3}-\mathrm{c}_{2} \mathrm{c}_{3} \mathrm{~s}_{1}\right)+\mathrm{c}_{4} \mathrm{~s}_{1} \mathrm{~s}_{2}\right)+$

$$
\mathrm{s}_{5}\left(\mathrm{c}_{4}\left(\mathrm{c}_{1} \mathrm{~s}_{3}-\mathrm{c}_{2} \mathrm{c}_{3} \mathrm{~s}_{1}\right)-\mathrm{s}_{4} \mathrm{~s}_{1} \mathrm{~s}_{2}\right)
$$

$o_{z}=\mathrm{c}_{5}\left(\mathrm{c}_{4} \mathrm{c}_{2}+\mathrm{c}_{3} \mathrm{~s}_{4} \mathrm{~s}_{2}\right)-\mathrm{s}_{5}\left(\mathrm{c}_{2} \mathrm{~s}_{4}-\mathrm{c}_{4} \mathrm{c}_{3} \mathrm{~s}_{2}\right)$

$a_{x}=\mathrm{c}_{3} \mathrm{~s}_{1}-\mathrm{c}_{1} \mathrm{c}_{2} \mathrm{~s}_{3}$

$a_{y}=-\mathrm{c}_{1} \mathrm{c}_{3}-\mathrm{c}_{2} \mathrm{~s}_{1} \mathrm{~s}_{3}$

$a_{z}=\mathrm{s}_{2} \mathrm{~s}_{3}$

$p_{x}=a_{4}\left(c_{4}\left(s_{1} s_{3}+c_{1} c_{2} c_{3}\right)+c_{1} s_{4} s_{2}\right)-d_{3} c_{1} s_{2}$

$p_{y}=-a_{4}\left(c_{4}\left(c_{1} s_{3}-c_{2} c_{3} s_{1}\right)-s_{4} s_{1} s_{2}\right)-d_{3} s_{1} s_{2}$

$p_{z}=a_{4}\left(\mathrm{c}_{2} \mathrm{~s}_{4}-\mathrm{c}_{4} \mathrm{c}_{3} \mathrm{~s}_{2}\right)-d_{1}-d_{3} c_{2}$

Note: $\mathrm{s}_{i}=\sin \theta_{i}, \quad \mathrm{c}_{i}=\cos \theta_{i}$.

The inverse kinematics solution is to solve the joint variables according to the position and pose of the end effector of the robot. That is to say, $n, o, a, p$ are known variables, and the joint variables $\theta_{1}, \theta_{2}, \theta_{3}, \theta_{4}, \theta_{5}$ are obtained. The inverse transformation matrix is multiplied by the two sides of the kinematic equation. We can separate the joint variables and solve them. The specific steps are as follows:
${ }_{1}^{0} T^{-1}\left(\theta_{1}\right)_{5}^{0} T={ }_{2}^{1} T\left(\theta_{2}\right){ }_{3}^{2} T\left(\theta_{3}\right){ }_{4}^{3} T\left(\theta_{4}\right){ }_{5}^{4} T\left(\theta_{5}\right)$

(2)

$\left[\begin{array}{cccc}c \theta_{1} & s \theta_{1} & 0 & 0 \\ -s \theta_{1} & c \theta_{1} & 0 & 0 \\ 0 & 0 & 1 & d_{1} \\ 0 & 0 & 0 & 1\end{array}\right]\left[\begin{array}{cccc}n_{x} & o_{x} & a_{x} & p_{x} \\ n_{y} & o_{y} & a_{y} & p_{y} \\ n_{z} & o_{z} & a_{z} & p_{z} \\ 0 & 0 & 0 & 1\end{array}\right]={ }_{5}^{1} T$

${ }_{5}^{0} T_{5}^{4} T^{-1}\left(\theta_{5}\right)={ }_{1}^{0} T\left(\theta_{1}\right) \frac{1}{2} T\left(\theta_{2}\right){ }_{3}^{2} \mathrm{~T}\left(\theta_{3}\right)_{4}^{3} \mathrm{~T}\left(\theta_{4}\right)$

$\left[\begin{array}{cccc}n_{x} & o_{x} & a_{x} & p_{x} \\ n_{y} & o_{y} & a_{y} & p_{y} \\ n_{z} & o_{z} & a_{z} & p_{z} \\ 0 & 0 & 0 & 1\end{array}\right]\left[\begin{array}{cccc}c \theta_{5} & s \theta_{5} & 0 & -a_{4} c \theta_{5} \\ -s \theta_{5} & c \theta_{5} & 0 & a_{4} s \theta_{5} \\ 0 & 0 & 1 & 0 \\ 0 & 0 & 0 & 1\end{array}\right]={ }_{4}^{0} T$

The corresponding elements in the matrix are equal.

$-a_{x} \mathrm{~s}_{1}+a_{y} c_{1}=-c_{3}$
$a_{x} c_{1}+a_{y} \mathrm{~s}_{1}=-\mathrm{c}_{2} \mathrm{~s}_{3}$
$a_{z}=\mathrm{s}_{2} \mathrm{~s}_{3}$
$-a_{4} n_{z} c_{5}+a_{4} o_{\mathrm{z}} s_{5}+p_{\mathrm{z}}=-d_{1}-d_{3} c_{2}$
$p_{z}=a_{4}\left(\mathrm{c}_{2} \mathrm{~s}_{4}-\mathrm{c}_{4} \mathrm{c}_{3} \mathrm{~s}_{2}\right)-d_{1}-d_{3} c_{2}$

From this $((4),(5),(6),(7),(8))$, we can solve the values of $\theta_{1}, \theta_{2}, \theta_{3}, \theta_{4}, \theta_{5}$.

\section{Simulation analysis}

A three-dimensional model of rehabilitation robot is established in Pro/E, and the size of simulation model is set according to the size of human upper limb. $d_{1}=$ $190 \mathrm{~mm}, d_{3}=300 \mathrm{~mm}, a_{4}=240 \mathrm{~mm}$. The threedimensional model is imported into ADAMS software [6]. We set the material properties of parts and add constraints in ADAMS. In order to enable the robot to achieve the desired trajectory motion, a driving function is added to each rotating pair to make the robot move under each driving function. The driving function is as follows:

Motion $1=$ STEP (time, $0.0, \quad 0.0 \mathrm{~d}, \quad 3.0, \quad-$ 30.0d)+STEP(time, 3.0, 0.0d, 9.0, 60.0d)+STEP(time, $9.0,0.0 \mathrm{~d}, 12.0,-30.0 \mathrm{~d})$.

Motion2=STEP(time, 42.0, 0.0d, 48.0, -60.0d )+STEP

(ti- me, 48.0, 0.0d, 51.0, 30.0d).

Motion3=STEP(time, 30.0,0.0d,39.0,90.0d)+STEP(time, 39.0,0.0d,63.0,0.0d)+STEP(time,63.0,0.0d,72.0,-90.0d).

Motion4=STEP(time, 12.0,0.0d,21.0,-90.0d)+STEP(time, $21.0,0.0 \mathrm{~d}, 30.0,90.0 \mathrm{~d})+\mathrm{STEP}($ time $, 48.0,0.0 \mathrm{~d}, 54.0,-90.0 \mathrm{~d})$ + STEP(time, 54.0, 0.0d, 63.0, 90.0d).

Motion5=STEP(time, 12.0,0.0d,21.0,-10.0d)+STEP(time, $21.0,0.0 \mathrm{~d}, 30.0,10.0 \mathrm{~d})+\mathrm{STEP}($ time $, 48.0,0.0 \mathrm{~d}, 54.0,-10.0 \mathrm{~d})$ + STEP(time,54.0,0.0d,63.0,10.0d).

The Marker points are set up in the end effector and moved under the driving function. The displacement simulation curves of Marker points in $\mathrm{X}, \mathrm{Y}$ and $\mathrm{Z}$ directi- ons are obtained, as shown in Figure 6. 


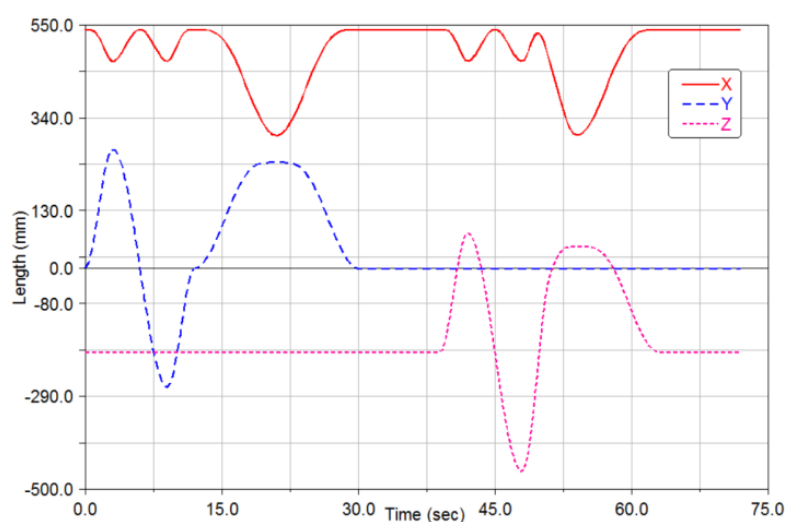

Figure 6. Displacement simulation curves of the end of the robot.

According to the forward kinematics equation, the range of the $\theta_{i}$ and the given trajectory motion, the displacement theoretical curves of the end effector in $X$, $\mathrm{Y}$ and $\mathrm{Z}$ directions are obtained by using MATLAB software, as shown in Figure 7.

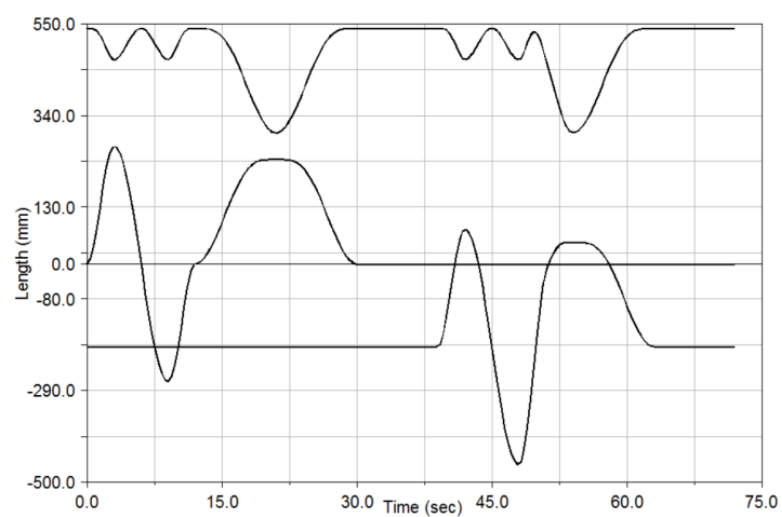

Figure 7. Displacement theory curves of the end of the robot.

It can be concluded that the theoretical results and simulation results are completely consistent, which proves the correctness of the kinematics equation.

As shown in Figure 8 and Figure 9, we can conclude that there is no mutation in the curve. The robot is stable in operation. It is consistent with the stability of patients during rehabilitation training. The result shows that the strategy is feasible.

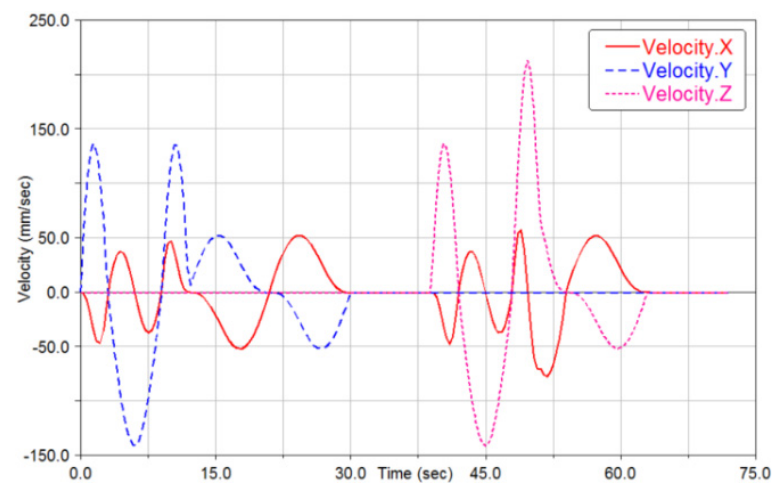

Figure 8. Velocity curves of the end of the robot.

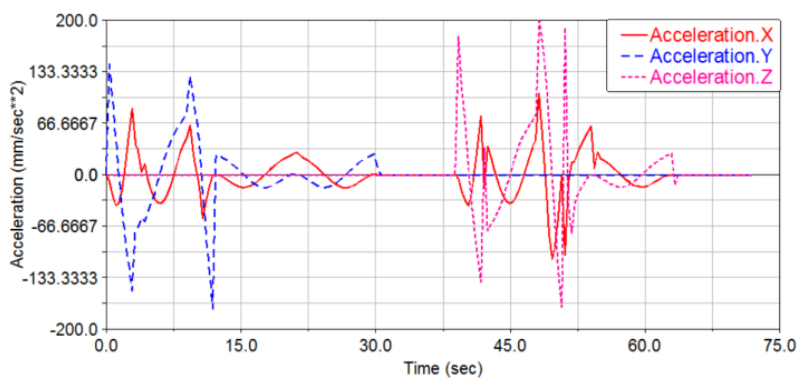

Figure 9. Acceleration curves of the end of the robot.

\section{Conclusion}

In this paper, the physiology of human upper limb is analysed. According to the physiological and kinematic characteristics of upper limb, a new type of 5-DOF upper limb rehabilitation robot is designed. The kinematics analysis and simulation analysis of the upper limb rehabilitation robot are carried out, which further certifies the correctness of the theoretical derivation and the feasibility of the scheme.

\section{References}

1. Yongfeng $\mathrm{Yi}$, Arm rehabilitation training robot structure design and control system research, D. Harbin. Harbin Engineering University. (2004)

2. Dongyan Wang, Qingling Li, Zhijiang Du. Study on exoskeletal rehabilitation robot for upper limb and its control method., J. Journal of Harbin Engineering University.(2007)

3. Mingxi Wang. anatomy of motion, M. Beijing, People's Physical Culture Publishing House,(2008)

4. E. J. McCormick, Ed., Human Factors Engineering, 3rd ed. New York:McGraw-Hill, (1970)

5. Zixing Cai. Robotics, M. Beijing, Tsinghua University Press, (2015)

6. Jianrong Zheng. The introduction and improve of virtual prototyping technology of ADAMS, M. Beijing, China Mechine Press, (2001) 